\title{
Intracholecystic Papillary Neoplasm Localized to the Cystic Duct: A Case Report
}

\author{
Koichi Oishi ${ }^{a}$ b $\quad$ Masahiro Ikeda ${ }^{a}$ Kazuhiro Toyota ${ }^{a}$ Koichi Mandai ${ }^{c}$ \\ Tadateru Takahashi ${ }^{a, d}$ \\ aDepartment of Surgery, National Hospital Organization Higashihiroshima Medical Center, \\ Higashihiroshima, Japan; b Department of Surgery, Chugoku Rosai Hospital, Kure, Japan; \\ 'Department of Pathology, National Hospital Organization Higashihiroshima Medical \\ Center, Higashihiroshima, Japan; ${ }^{\mathrm{d} D e p a r t m e n t}$ of Gastroenterological and Transplant \\ Surgery, Applied Life Sciences, Institute of Biomedical and Health Sciences, Hiroshima \\ University, Hiroshima, Japan
}

\section{Keywords}

Intracholecystic papillary neoplasm · Cystic duct · Gallbladder

\begin{abstract}
Intracholecystic papillary neoplasm (ICPN) of the gallbladder is a premalignant lesion. An ICPN arising from the cystic duct is rare. A woman in her 60 s exhibited dilatation of the common bile duct on computed tomography (CT) performed for screening of respiratory disease. The $\mathrm{CT}$ revealed an enhancing mass, $3.3 \mathrm{~cm}$ in diameter, in the cystic duct. Endoscopic ultrasonography showed a well-demarcated, hyperechoic mass in the dilated cystic duct. Endoscopic retrograde cholangiography showed that the common bile duct was slightly retracted by the dilated cystic duct. Cytological analysis of the bile juice did not show any evidence of malignancy. She was diagnosed with a cystic bile duct tumor suggestive of ICPN. Cholecystectomy, resection of the extrahepatic bile duct, and lymph node dissection were performed. Macroscopically, the papillary-proliferated tumor was localized to the cystic duct. No critical lesions were evident in the common bile duct. Histologically, the tumor in the cystic duct showed intraluminal growth with a papillary configuration without malignancy. Based on these findings, the tumor was diagnosed as an ICPN. We encountered a rare case of ICPN localized to the cystic duct of the gallbladder.
\end{abstract}




\section{Introduction}

Intracholecystic papillary neoplasm (ICPN) was first documented as a premalignant lesion of the gallbladder in the World Health Organization (WHO) classification of tumors of the gallbladder and extrahepatic bile ducts published in 2010 [1]. In 2012, Adsay et al. [2] proposed the term intracholecystic papillary-tubular neoplasm for a precancerous lesion of the gallbladder with papillary or tubular growth patterns. In 2019, WHO classified benign epithelial tumors and precursors of the gallbladder and extrahepatic bile ducts as adenoma, biliary intraepithelial neoplasia, ICPN, and intraductal papillary neoplasm of the bile duct [3]. ICPNs have been defined recently and are rare. In the biliary system, the cystic duct is included in the gallbladder and not in the extrahepatic bile duct. Moreover, an ICPN located in the cystic duct of the gallbladder is extremely rare. Herein, we report a case of ICPN arising from the cystic duct.

\section{Case Presentation}

A female patient in her 60s visited a clinic regularly. She had rheumatoid arthritis, hypertension, and an ovarian cyst. Dilatation of the common bile duct was incidentally detected on computed tomography performed to screen for respiratory disease. Therefore, she was referred to our hospital for further examination. Laboratory findings were within the reference range, except for slight elevation in the levels of alkaline phosphatase, aspartate aminotransferase, alanine aminotransferase, and C-reactive protein. The levels of tumor markers such as carcinoembryonic antigen and carbohydrate antigen 19-9 were within the reference range. Computed tomography revealed an enhancing mass, $3.3 \mathrm{~cm}$ in diameter, in the cystic duct of the gallbladder (Fig. 1a, b). The mass in the cystic duct of the gallbladder exhibited a low-intensity signal on T1-weighted imaging (Fig. 1c), a slight high-intensity signal on T2-weighted imaging (Fig. 1d), and a high-intensity signal on diffusion-weighted imaging (Fig. 1e). Magnetic resonance cholangiopancreatography showed a tumor-like defect in the dilated cystic duct (Fig. 1f). Positron emission tomography revealed localized accumulation of fludeoxyglucose $(4.2 \mathrm{~F})$ in the cystic duct tumor (Fig. 2a). Endoscopic ultrasonography showed a well-demarcated, hyperechoic mass in the dilated cystic duct. The common bile duct showed no abnormalities other than slight dilatation (Fig. 2b). Endoscopic retrograde cholangiography (ERC) showed that the common bile duct was slightly retracted by the dilated cystic duct (Fig. 2c). Cytological analysis of the bile juice did not show any evidence of malignancy. Based on these findings, she was diagnosed with a cystic bile duct tumor, suggestive of ICPN. However, considering the malignant nature and extent of spread of the tumor to the junction of the common bile duct, we planned and performed cholecystectomy, resection of the extrahepatic bile duct, and lymph node dissection. Frozen sections of the stump of the common bile duct did not show any evidence of malignancy. Macroscopically, the papillary-proliferated tumor was localized to the cystic duct (Fig. 3a). No critical lesions were noticed in the common bile duct. Histologically, the tumor in the cystic duct showed intraluminal growth with a papillary configuration and was noninvasive (Fig. 3b, c). Therefore, this tumor was diagnosed as ICPN with low-grade intraepithelial neoplasia because of the localization of the gallbladder lesion to the cystic duct. On immunohistochemical examination (Fig. $3 \mathrm{~d}-\mathrm{g}$ ), the tumor cells of ICPN localized to the cystic duct reacted with MUC-5AC and MUC-6, reacted slightly with MUC-2 but did not react with MUC-1. The tumor showed gastric morphology, typically resembling gastric foveolar epithelium with elongated interconnecting glands lined by tall columnar cells. Therefore, the histological pattern of ICPN was classified as gastric foveolar type.

The patient was discharged 9 days after surgery without any complications. She had no recurrence for 2 years after the resection.

\section{Karger'}



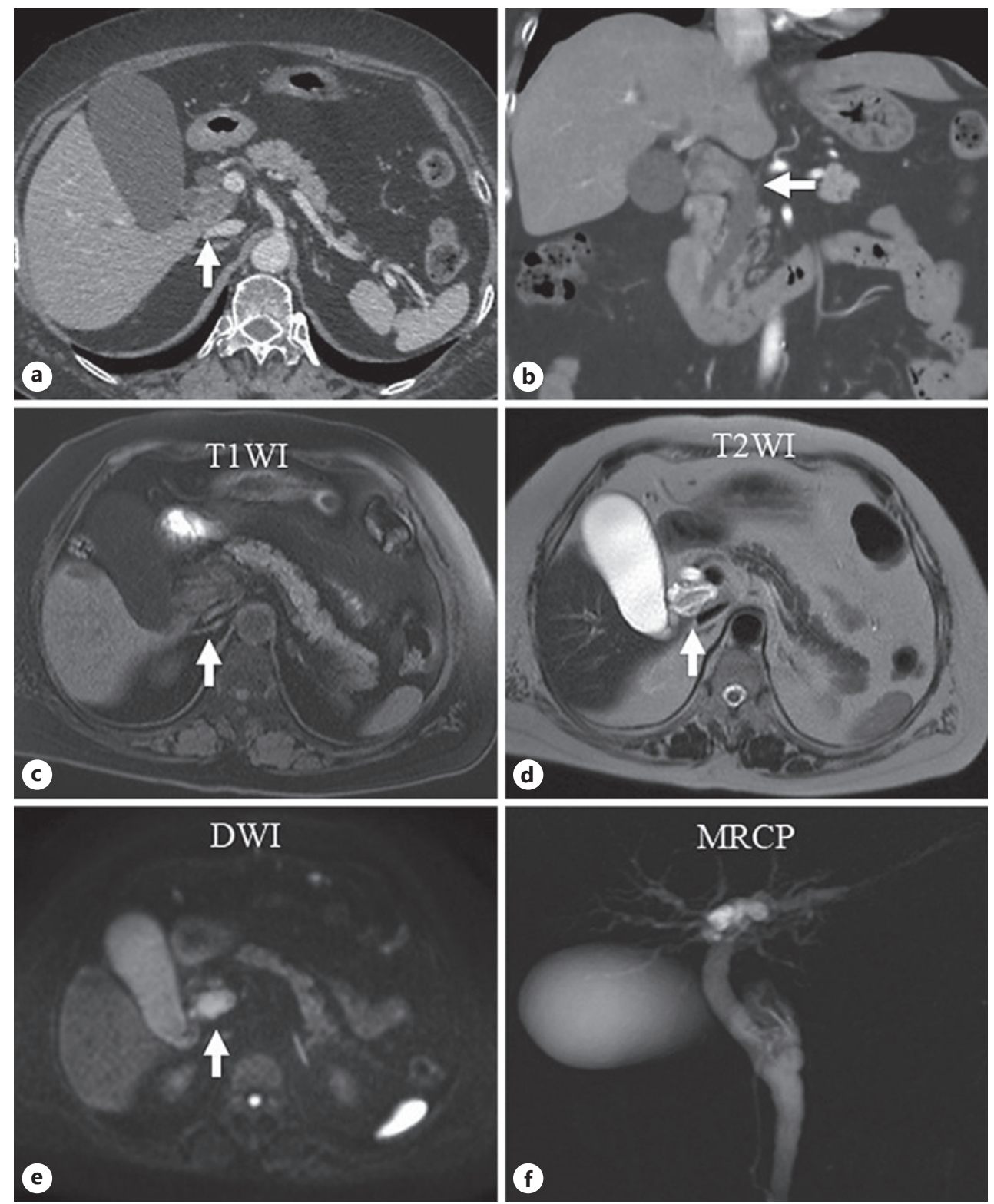

Fig. 1. CT with contrast enhancement. The arrows indicate a tumor in the cystic duct. a Axial image. b Coronal image. Magnetic resonance imaging. The mass in the cystic duct reveals a low-intensity signal on T1-weighted imaging (c), a slight high-intensity signal on T2-weighted imaging (d), and a high-intensity signal on diffusionweighed imaging (e). Magnetic resonance cholangiopancreatography (f) shows tumor-like defect in the dilated cystic duct.

\section{Discussion}

In 2010, WHO classification of tumors of the gallbladder classified precursors and early lesions of the gallbladder cancer as ICPN [1]. Papillary lesions involving the gallbladder are referred to as ICPN. ICPN is defined as a grossly visible, mass-forming, noninvasive, epithelial neoplasm arising in the mucosa, and projecting into the lumen of the gallbladder [3].

The mean age of patients with ICPN in most reports is 60 years [2, 4-6]. The majority of patients are females, with a female:male ratio of $3: 1$ to $6: 1$ [2, 5-7]. Approximately $50 \%$ 


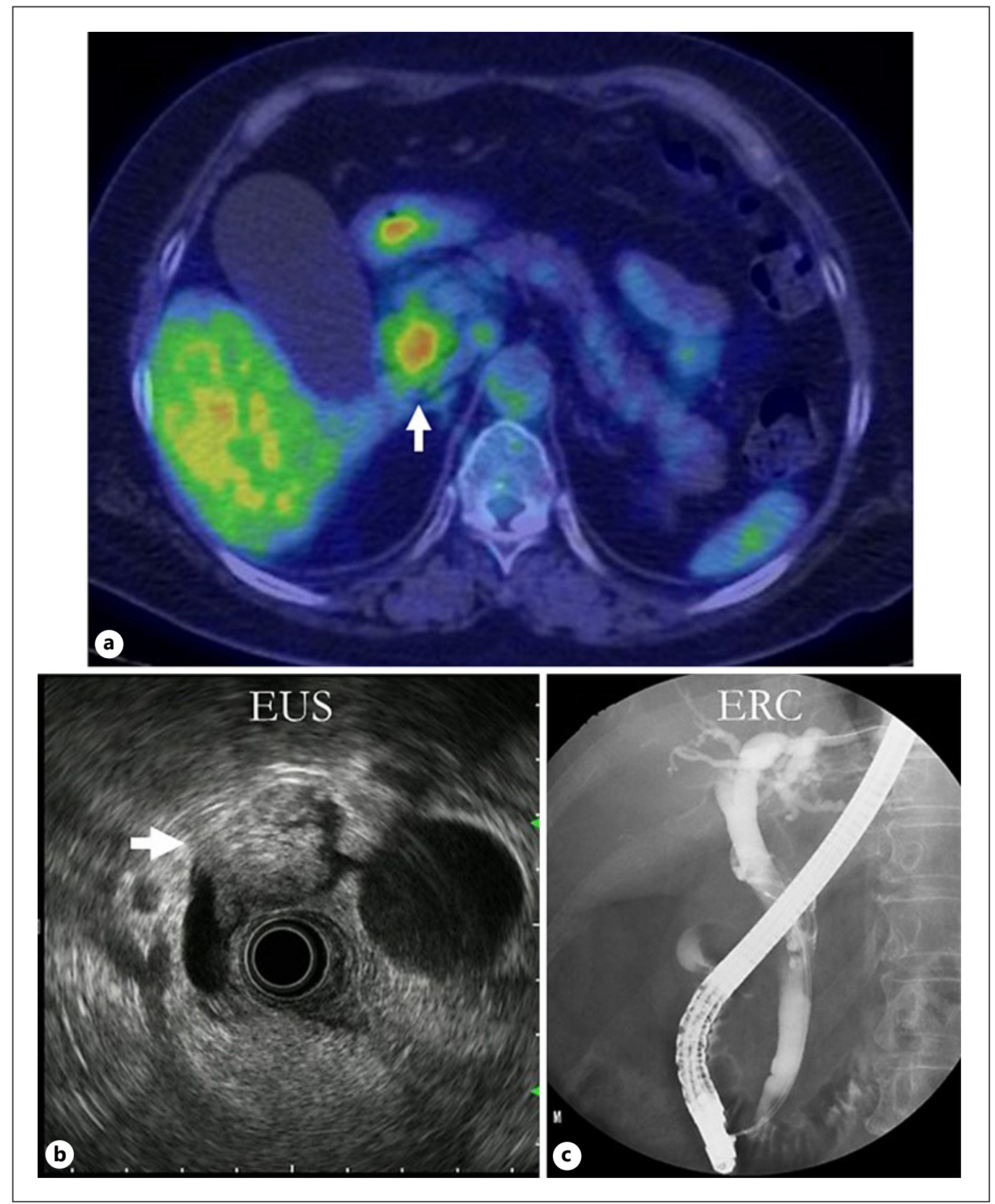

Fig. 2. a Positron emission tomography shows localized accumulation of fludeoxyglucose (4.2 F) in the mass in the cystic duct (arrow). b Endoscopic ultrasonography shows a well-demarcated, hyperechoic mass in the dilated cystic duct (arrow). The common bile duct shows no abnormalities other than slight dilatation. c Endoscopic retrograde cholangiography shows slight retraction of the common bile duct by the dilated cystic duct.

of patients with ICPN present with pain in the right upper quadrant of the abdomen. The remaining $50 \%$ of the patients are asymptomatic [2]. This case was of a woman in her $60 \mathrm{~s}$ who was asymptomatic. Approximately one-third of ICPNs are multifocal. The median tumor size is $2.2 \mathrm{~cm}$ [2]. Dilatation of the bile ducts, and sometimes, cystic dilatations are observed. Hypersecretion or luminal accumulation of mucin may occasionally occur. In this case, ERC showed only defects caused by the tumor without any mucinous formations. 


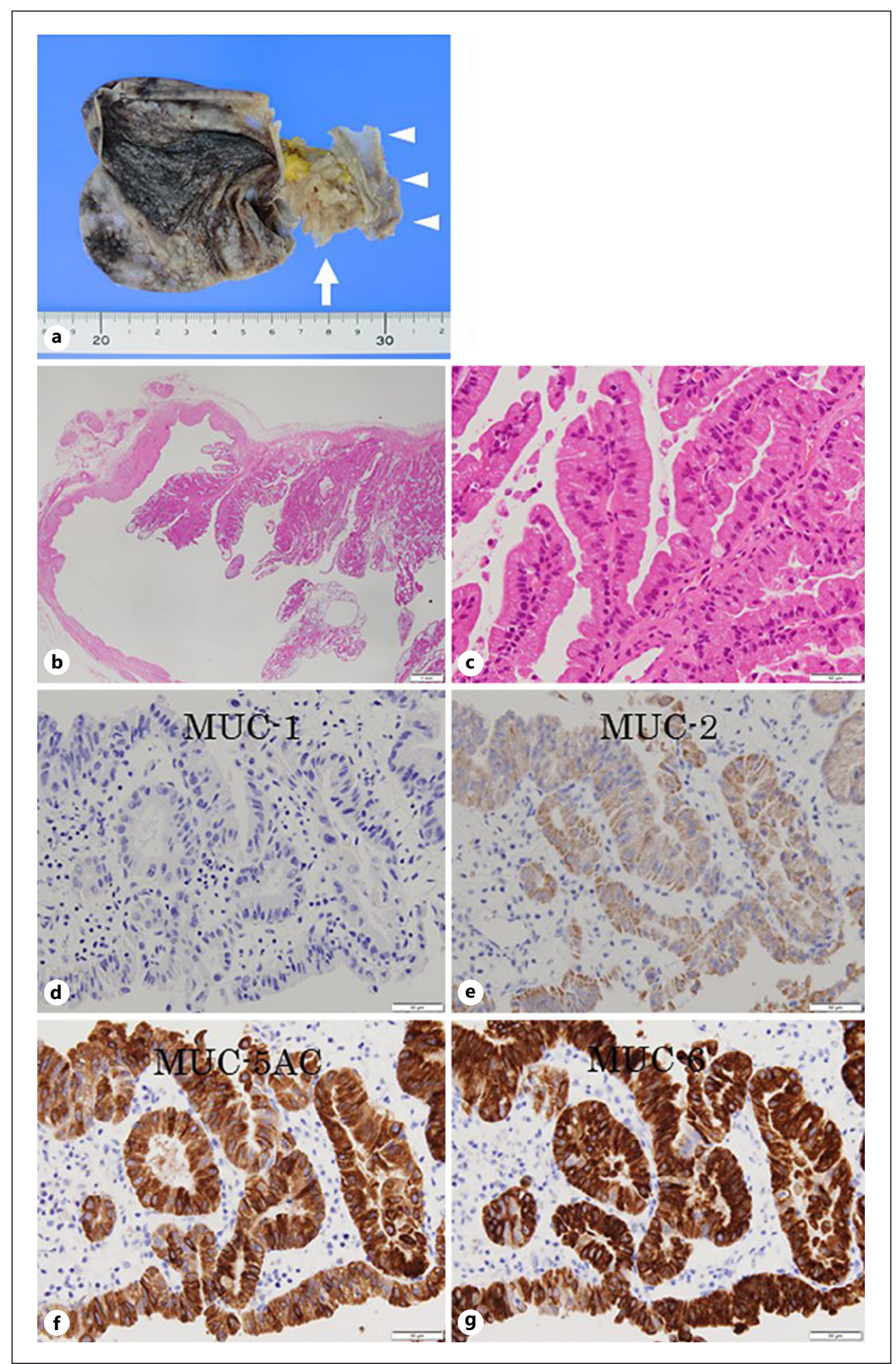

Fig. 3. a Gross feature of ICPN. The papillary-proliferated tumor localized in the cystic duct (arrow). No critical lesion in the common bile duct (arrowhead). Microscopic features of ICPN. b Low power examination. H\&E staining, c high power examination. H\&E staining. The tumor in the cystic duct shows irregular papillary proliferation with a fern-leaf-like structure, and it is noninvasive. Immunohistochemical findings of ICPN. d MUC-1. e MUC-2.f MUC-5AC. g MUC-6. H\&E, hematoxylin and eosin. 
According to the degree of atypia in the epithelium, ICPNs are classified as low-grade lesions (mild to moderate atypia) or high-grade lesions (noninvasive papillary carcinoma). Four phenotypes of ICPNs are histologically and immunohistochemically recognized: biliary, gastric, intestinal, and oncocytic types [3]. Gastric ICPNs comprise two subtypes: gastric foveolar and gastric pyloric types. In the gastric foveolar type, tall columnar cells of the gastric region are diffusely positive for MUC-5AC, while some may express MUC-6 [2, 8]. In the gastric pyloric type, smaller tubular glands are diffusely and strongly positive for MUC-6 [2]. In our case, microscopically, there were no destructive findings. While the tumor had diffuse and strong co-immunoreactivity for both MUC-5AC and MUC-6, the tumor showed a gastric morphology, typically resembling the gastric foveolar epithelium with elongated interconnecting glands lined by tall columnar cells containing abundant mucin. Therefore, the tumor in our patient was diagnosed as ICPN of gastric foveolar type with low-grade intraepithelial neoplasia.

Although the ICPN of the cystic duct was diagnosed preoperatively, considering the possibility of malignant transformation, we performed cholecystectomy with resection of the extrahepatic bile duct and lymph node dissection. Pathological examination did not reveal any malignant lesions. However, histological analysis of the frozen sections showed borderline atypia. As it is difficult to diagnose malignant lesions intraoperatively using frozen sections, we believe that the choice of the surgical procedure was appropriate. According to the UICC TNM classification, cystic duct cancer is categorized as the gallbladder cancer. Therefore, this case was diagnosed as ICPN because of the presence of a gallbladder lesion, and not intraductal papillary neoplasm of the bile duct.

Some studies have reported cases of ICPN originating from the cystic duct [9-11]. However, these cases include tumors that involved the common bile duct and were not localized to the cystic duct. To the best of our knowledge, this is the first report of ICPN localized to the cystic duct. In conclusion, we report an extremely rare case of ICPN localized to the cystic duct of the gallbladder.

\section{Acknowledgment}

We thank Editage (www.editage.com) for the English language editing.

\section{Statement of Ethics}

This case report was exempted from ethical approval by the Institutional Review Board of the National Hospital Organization Higashihiroshima Medical Center. Written informed consent was obtained from the patient for performing the surgical procedures and the publication of this case report and related images.

\section{Conflict of Interest Statement}

The authors have no conflicts of interest to declare.

\section{Funding Sources}

The authors declare that there were no funding sources for this study.

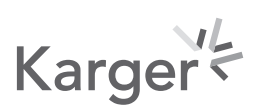




\section{Author Contributions}

K.O. drafted the manuscript. K.O. and T.T. reviewed and edited the manuscript. K.O., M.I., and K.T. participated in patient care. K.M. diagnosed the case pathologically. All authors read and approved the final manuscript.

\section{Data Availability Statement}

All data analyzed during this study are included in this article. Further inquiries can be directed to the corresponding author.

\section{References}

1 Albores-Saavedra J, Adsay NV, Crawford JM, Klimstra DS, Kloppel G, Sripa B, et al. Carcinoma of the gallbladder and extrahepatic bile ducts. In: Bosman FT, Carneiro F, Hruban RH, Theise ND, editors. WHO classification of tumours of the digestive system. 4th ed. Lyon: International Agency for Research on Cancer; 2010. p. 266-74.

2 Adsay V, Jang KT, Roa JC, Dursun N, Ohike N, Bagci P, et al. Intracholecystic papillary-tubular neoplasms (ICPN) of the gallbladder (neoplastic polyps, adenomas, and papillary neoplasms that are $\geq 1.0 \mathrm{~cm}$ ): clinicopathologic and immunohistochemical analysis of 123 cases. Am J Surg Pathol. 2012 Sep;36(9):1279-301.

3 Basturk O, Aishima S, Esposito I. Intracholecystic papillary neoplasm. In: WHO classification of tumours editorial board: digestive system tumours (WHO classification of tumours). 5th ed. Lyon: International Agency for Research on Cancer; 2019. p. 276-8.

4 Isozaki M, Ohike N, Tajiri T, Mitsuya T, Takimoto M. Clinicopathological study of intracholecystic papillarytubular neoplasms (ICPNs) of the Gallbladder. Showa Univ J Med Sci. 2014;26(1):17-26.

5 Bennett S, Marginean EC, Paquin-Gobeil M, Wasserman J, Weaver J, Mimeault R, et al. Clinical and pathological features of intraductal papillary neoplasm of the biliary tract and gallbladder. HPB. 2015 Sep;17(9):811-8.

6 Argon A, Barbet FY, Nart D. The relationship between intracholecystic papillary-tubular neoplasms and invasive carcinoma of the gallbladder. Int J Surg Pathol. 2016 Sep;24(6):504-11.

7 Hazarika P, Sharma MK. Intracholecystic papillary-tubular neoplasm of gallbladder: a 5-year retrospective pathological study. Indian J Pathol Microbiol. 2018 Oct;61(4):516-9.

8 Albores-Saavedra J, Chablé-Montero F, González-Romo MA, Ramírez Jaramillo M, Henson DE. Adenomas of the gallbladder. Morphologic features, expression of gastric and intestinal mucins, and incidence of high-grade dysplasia/carcinoma in situ and invasive carcinoma. Hum Pathol. 2012 Sep;43(9):1506-13.

9 Kadowaki Y, Yokota Y, Komoto S, Kubota N, Okamoto T, Ishido N, et al. Spread of intraductal papillary neoplasm arising from the cystic duct of the biliary tree. Case Rep Gastroenterol. 2016;10(1):7-16.

10 Fujii Y, Noda Y, Koshita S, Kanno Y, Ogawa T, Kusunose H, et al. Intracholecystic papillary-tubular neoplasm of the gallbladder originating in the cystic duct with extensive intraepithelial progress in the common bile duct. Clin J Gastroenterol. 2019 Jun;12(3):197-204.

11 Iseki M, Mizuma M, Aoki Y, Aoki S, Hata T, Takadate T, et al. Intracholecystic papillary neoplasm arising in the cystic duct and extending into common bile duct: a case report. Clin J Gastroenterol. 2021 Apr;14(2):668-77. 